\title{
ARTICLE
}

\section{Increased subjective and reinforcing effects of initial nicotine exposure in young adults with attention deficit hyperactivity disorder (ADHD) compared to matched peers: results from an experimental model of first-time tobacco use}

\author{
Scott H. Kollins ${ }^{1}$, Maggie M. Sweitzer ${ }^{1}$, F. Joseph McClernon ${ }^{1}$ and Kenneth A. Perkins ${ }^{2}$
}

Individuals with attention deficit hyperactivity disorder (ADHD) are at increased risk for adverse cigarette smoking outcomes, and little is known about factors underlying this risk. This study sought to evaluate the effects of initial nicotine exposure in young adults with and without ADHD using a novel paradigm of exposure to model initial smoking experiences. Participants were young adult nonsmokers ( $n=61$ ADHD, $n=75$ Control) between the ages of 18-25 years (inclusive) who reported never having smoked a full cigarette, and no tobacco use in the prior 3 years. Participants were exposed to three different blinded doses of intranasally administered nicotine $(0,0.5,1.0 \mathrm{mg})$ across three separate fixed dose experimental sessions. In subsequent sessions, participants were given the opportunity to self-administer nicotine under two different conditions-high and low cognitive demand. Physiological, subjective, and reinforcing effects of nicotine were the main outcomes. Nicotine plasma levels, and no group differences in effects of nicotine on heart rate or blood pressure, confirmed comparable dosing exposure across groups. ADHD participants reported significantly greater dizziness following nicotine, and greater pleasant subjective effects across all conditions, compared to non-ADHD non-smokers. There were no group differences on subjective reports of bad or unpleasant effects. Subsequent nicotine self-administration was significantly higher among non-smokers with ADHD, and their choices of nicotine were not influenced by cognitive condition. There are meaningful differences between young adults with and without ADHD with respect to the initial subjective and reinforcing effects of nicotine; and interventions to prevent use should start prior to typical age of experimentation among ADHD patients.

Neuropsychopharmacology (2020) 45:851-856; https://doi.org/10.1038/s41386-019-0581-7

\section{INTRODUCTION}

Attention deficit hyperactivity disorder (ADHD) affects millions of individuals and is a significant risk factor for smoking [1-5]. Individuals with ADHD start smoking earlier and become more dependent $[5,6]$. Moreover, individuals with ADHD are more likely to progress from smoking experimentation to regular use [7]. Given the associated morbidity and mortality, clarifying factors that increase risk for smoking in ADHD patients is an important public health challenge and critical to help prevent and treat smoking in this high-risk group.

Mechanisms underlying risk of smoking in ADHD are relatively underexplored. Regular smokers with ADHD have been shown to work more for cigarette puffs than non-ADHD smokers, suggesting that the reinforcing effects of smoking may be higher in this group [8]. Smokers with ADHD (compared to non-ADHD) show more severe symptoms of nicotine withdrawal, including disrupted inhibitory control, and these differences are associated with differential activation in occipital/parietal regions [9-11]. These studies, however, have been conducted in individuals who are already regular smokers. No studies have prospectively evaluated factors that might confer risk for smoking in an ADHD sample.
The primary objective of the present study was to determine whether initial experiences with nicotine differed for young adult non-smokers with ADHD compared to those without. We used a validated approach for modeling initial exposure to nicotine, without the non-nicotine constituents in tobacco smoke, using nicotine nasal spray. This procedure allows for assessment of acute effects of measured doses of nicotine in a less invasive manner than intravenous administration and more closely approximates the pharmacokinetics of actual smoking compared to other forms of administration (e.g., transdermal) [12]. This paradigm has been shown to be sensitive to individual differences in response to nicotine: current smokers more readily administer nicotine nasal spray than ex-smokers or non-smokers [13] and aspects of impulsivity - a core component of ADHD-were associated with greater self-administration among nonsmoking males [14]. We hypothesized that the effects of initial exposure to nicotine nasal spray would be different in nonsmokers with ADHD compared to non-ADHD peers. We hypothesized that non-smokers with ADHD would report more positive and fewer negative subjective effects of initial experiences with nicotine and that they would be more likely to self-administer nicotine. Given that nicotine is

\footnotetext{
${ }^{1}$ Department of Psychiatry \& Behavioral Sciences, Duke University School of Medicine, Durham, NC, USA and ${ }^{2}$ Departments of Psychiatry, Psychology, and Epidemiology, University of Pittsburgh, Pittsburgh, PA, USA

Correspondence: Scott H. Kollins (scott.kollins@duke.edu)
}

Received: 16 August 2019 Revised: 3 November 2019 Accepted: 23 November 2019

Published online: 30 November 2019 
Table 1. Study inclusion/exclusion criteria.

\begin{tabular}{|c|c|}
\hline Inclusion criteria & Exclusion criteria \\
\hline
\end{tabular}

known to influence neurocognitive processes implicated in ADHD (i.e., attention, inhibitory control) [15], we also examined self-administration of nicotine under two different cognitive conditions-a high demand and a low demand condition-and hypothesized that the ADHD participants would be more likely to self-administer nicotine under high demand cognitive conditions.

\section{METHODS}

This study was reviewed and approved by the local IRB and the study was conducted consistent with all guidelines pertaining to the ethical conduct of human research.

\section{Participants/setting}

Participants for this study were 136 ( $n=61$ ADHD, $n=75$ Control) young adult nonsmokers recruited between January 2013 and September 2017 from an ADHD clinic and the community through direct referrals, advertisements, and word-of-mouth. Inclusion criteria for the study included healthy males and females between the ages of 18-25 years (inclusive) who reported never having smoked a full cigarette or used other nicotine-containing products, and no tobacco use in the prior 3 years. Participants in the ADHD group met full criteria for ADHD, any subtype as determined by standardized diagnostic procedures (Supplementary Material); other DSM diagnoses or current treatment were exclusionary. ADHD participants currently treated with stimulant medication $(n=19)$ agreed to discontinue their medication for $72 \mathrm{~h}$ prior to each experimental session. Complete inclusion/ exclusion criteria are listed in Table 1.

Design/procedures

Participants completed six sessions: 1 Screening session to assess eligibility; three Fixed Dose sessions (FDS) wherein they were exposed to three different doses of nicotine; and two Choice Session (CS) wherein participants could choose between nicotine and placebo nasal spray.

During screening visits, informed consent was obtained and a breath sample taken to assess expired-air $\mathrm{CO}$ and ensure no recent smoking $(\mathrm{CO}<3 \mathrm{ppm})$ [16]. Saliva, blood, and urine samples were collected to assess (1) plasma cotinine levels to verify no recent nicotine exposure $(<5 \mathrm{ng} / \mathrm{ml})$ : (2) illicit drug use; and (3) pregnancy in females. Breath alcohol level (BAL) of 0.0 was required to continue screening and all subsequent study visits. A smoking history was gathered to ensure potential subjects met the lifetime tobacco use criterion, and collateral reports from three sources (e.g. friends, family) were used to verify self-reports of nonsmoking status. A medical history/physical exam was also conducted by the study physician.

Standardized diagnostic procedures were followed to assess eligibility for both groups (ADHD and non-ADHD) in the study. Procedures included the administration of the Structured Clinical Interview for DSM (SCID) [17]; the Conners Adult ADHD Diagnostic Interview (CAADID) [18]; and the use of the Conners Adult ADHD Rating Scales [19]. These measures were administered and reviewed by a qualified and trained clinician (Ph.D. level psychologist or psychiatrist M.D.), along with a clinical interview with the patients. The CAADID was used to confirm diagnosis in the ADHD group and to ensure that those in the non-ADHD group did not meet criteria. The SCID and the clinical interview was used to rule out the presence of exclusionary DSM psychiatric diagnoses other than ADHD. Eligible participants completed 3 FDS followed by 2 CS. Dosing order for FDS and cognitive demand condition for CS was counterbalanced across participants. At the beginning of each FDS/CS, participants provided a urine sample to test for illegal drugs and to confirm abstinence from nicotine. BAL was also assessed.

Each FDS was identical except for the dose of nicotine evaluated. Vital signs, subjective, mood and performance measures were collected. This sequence of assessments was repeated twice to obtain a stable baseline and to habituate participants to the testing environment. The same assessment sequence was then repeated three more times following nasal spray administration, with each sequence separated by $45 \mathrm{~min}$. We selected this inter-trial interval $(45 \mathrm{~min})$ to reduce likelihood of adverse events, since previous studies have used a shorter interval (e.g., $30 \mathrm{~min}$ ) [14]. Nicotine and placebo were administered intranasally via standardized procedures. During each dosing trial, participants received two sprays of nasal spray. For the $0 \mathrm{mg}$ session, both sprays were placebo; for the $0.5 \mathrm{mg}$ session, one spray each was 
placebo and nicotine; and for the $1 \mathrm{mg}$ session, both sprays were nicotine. Participants were blinded to which drug they were receiving. Research staff were also blinded, but were instructed by unblinded study staff on which spray bottles to use. Plasma nicotine concentrations to verify dosing and examine individual differences, were obtained via venipuncture, after the last dosing trial for each of the three FDS.

During CS, each participant completed 2 "sampling" trials (1 each of $1 \mathrm{mg}$ nicotine and placebo, identified by letter code) separated by $45 \mathrm{~min}$. These were to ensure that participants could identify which letter code was associated with acute drug effects, since conditions were blinded, and in order to provide a basis for the subsequent choice procedure. Following each of the two sampling trials, participants were exposed to abbreviated versions of the cognitive conditions for that day (high demand versus low demand condition). Choice procedures began 45 min after the second sampling trial and a series of five choice trials were administered, with 45 min between trials. For each trial, participants were given the opportunity to administer two sprays from one or both bottles as described above, for a total of 10 choices.

In the high cognitive demand (HD) condition, subjects completed a mathematical vigilance task following each choice trial. During this 10-min task, participants were given a 5-page set of math problems ( 80 problems/page) and instructed to work as many problems as possible in the allotted time. The level of difficulty was calibrated for each participant during screening, and designed to result in completion of $10-15$ problems $/ \mathrm{min}$. Participants received $\$ 0.50$ for every 15 problems that they accurately completed. Subjective effects and vital signs were also collected and during the intervening periods, participants were otherwise allowed to engage in a range of sedentary activities, including reading magazines or watching television until the next choice trial.

In the low cognitive demand (LD) condition, subjective effects measures and vital signs were similarly collected, and participants were otherwise free to participate in sedentary activities for the remaining time between trials. Periodically throughout the intervening time during the LD session, a tone sounded from a computer in the laboratory, indicating the non-contingent delivery of $\$ 0.50$. Money was delivered 10 times throughout each 45-min time period at randomly scheduled intervals to match the contingent payment that occurred under the HD condition as part of the mathematical vigilance task.

\section{Outcomes}

Physiological. Heart rate and blood pressure were measured prior to any drug administration and periodically throughout each session.

Subjective effects. A series of Visual Analog Scales (VAS) was presented on a computer screen and rated on a scale from 0 ("not at all") to 100 ("extremely") and assessed a range of subjective drug effects [20]. An Initial Reactions Questionnaire was also administered at the end of each of the FDS. This measure has been used widely in retrospective research on the effects of early experiences to smoking and yields three empirically derived factors: pleasant reactions, unpleasant reactions, and dizziness [21-23].

Nicotine reinforcement. During CS, response for nicotine during each trial was dichotomized to indicate whether any nicotine was selected ( 1 or 2 sprays from the nicotine bottle- $38 \%$ of all choices; compared with 2 sprays from the placebo bottle- $62 \%$ of all choices). [24] Choice for nicotine across each of the five trials within each session served as the primary measure of reinforcement (see Supplementary Table S3 for additional details of choice distributions across trials and sessions).
Study medications

Nicotine nasal spray (Nicotrol ${ }^{\oplus} ; 0.5 \mathrm{mg} / \mathrm{spray}$ ) was used for this study. The placebo consisted of $15 \mathrm{ml}$ sterile normal saline, $5 \mu \mathrm{l}$ of food grade capsaicin oil (Pure Cap extract) explicitly to match the acute nasal sensations from the Nicotrol spray and aid blinding, and $5 \mu \mathrm{l}$ of polysorbate $80 \mathrm{NF}$ to ensure stability of the solution. The spray bottles utilized a Preservative Free Pump-Nasal (PFP-N) from MeadWestvaco. Nicotine and placebo sprays were prepared by a local compounding pharmacy facility.

\section{Safety monitoring}

A number of steps were taken with this protocol to ensure safety of participants, particularly with respect to risk for smoking. First, at both Screening and following all Experimental Sessions, participants were asked how difficult they felt it would be to refrain from smoking over the next year. At Screening, answering this question with the following answers "Not Sure," "Difficult," or "Very Difficult" was exclusionary. Per protocol, if participants answered this question at the end of the study with the aforementioned responses, they would be scheduled with a clinician to discuss how to mitigate the possibility of smoking. No participants were excluded for this reason, and none indicated uncertainty about remaining a non-smoker at the end of the study. Second, all participants were queried 1 and 6 months after their final session to determine smoking status. No participants reported that they had initiated smoking following the study.

Finally, as part of an IND that was filed and FDA approved for the use of nicotine nasal spray in this study, additional safety monitoring was implemented to assess specific pulmonary health/ adverse effects at the 30-day follow-up contact (see Supplementary Material). No participants reported pulmonary adverse events that were deemed by the study physician to be related to the nicotine (or placebo) nasal sprays.

Statistical analysis

Statistical analyses used regression models, with distribution specified as normal for continuous outcomes during FDS and binomial for dichotomous choice outcomes during CS. Analyses were conducted using generalized estimating equations (GEE) in SAS 9.4 PROC GENMOD, which adjusts standard errors of parameter estimates using robust "sandwich" variance estimates to account for repeated observations [25]. GEE models are flexible with respect to outcome distribution, easily accommodating both normal and binary outcomes, and have been shown to produce unbiased estimates given sufficient sample sizes, as included here [26]. Covariance matrix specified as exchangeable in all analyses. All models were adjusted for age, sex, race (white vs. non-white). For FDS, nasal spray Dose (0, 0.5 , or $1.0 \mathrm{mg}$ nicotine), Group (ADHD and Control), and their interaction were entered as predictors for all models, with FDS order included as covariate. When appropriate, a two-level factor of Time (Pre-drug assessments 1 and 2 vs. post-drug assessments 3 through 5) was also included, along with linear effects of the five repeated assessments within each session. To ensure overall nicotine exposure levels were comparable across groups in FDS, we first applied these models to the plasma nicotine values assessed at the end of each FDS. We then examined additional physiological measures (e.g., heart rate, blood pressure), followed by subjective reactions. Simple effects were examined to explore significant interactions. Of note, given the linear increase in nicotine nasal spray content across the three FDS, Dose was considered as a non-categorical factor in primary analyses. This approach results in single parameter estimates for Dose effects, thereby simplifying interpretation of complex interactions. However, in cases of significant main effects of Dose, follow-up categorical models were used to examine pairwise comparisons between individual session doses. For CS, Group (ADHD and Control), Cognitive Demand Condition (HD and LD), and their interaction were included as predictors, and CS order 
and linear effects of repeated choice trials ( 3 through 7 within each session) were included as covariates.

\section{RESULTS}

Figure 1 illustrates the disposition of participants throughout the Recruitment and Screening portions of the study and Table 2 illustrates the baseline/demographic characteristics of participants who completed the study.

\section{Nicotine exposure/physiological effects}

Mean values for physiological measures across session and group are presented in Supplementary Table S1. As intended, these data indicate consistent exposure/bioavailability and physiological effects of the different nicotine doses across groups, as there were significant dose-dependent increases in plasma nicotine levels across FDS $(B=4.0 ; p<0.0001)$ but no effects of group or group $\times$ dose interaction. Pairwise contrasts indicated a significant increase in plasma nicotine levels at the $0.5 \mathrm{mg}$ dose relative to placebo $(B=1.6, p<0.0001)$ and at $1.0 \mathrm{mg}$ compared with $0.5 \mathrm{mg}$ $(B=2.4, p<0.0001)$. Nicotine also dose-dependently increased systolic $(B=5.9, p<0.0001)$ and diastolic $(B=7.1, p<0.0001)$ blood pressure and heart rate $(B=7.8, p<0.0001)$, as evidenced by significant dose $\times$ time interactions, but there were no main effects of group for any physiological measure. As with plasma nicotine levels, all measures were increased at the $0.5 \mathrm{mg}$ dose relative to placebo (all $p^{\prime} \mathrm{s}<0.01$, and at the $1.0 \mathrm{mg}$ dose compared with $0.5 \mathrm{mg}$ (all $p^{\prime} \mathrm{s}<0.01$ ). Dose $\times$ time $\times$ group interactions were significant for heart rate $(B=3.5, p<0.05)$ and diastolic blood pressure $(B=3.0, p<0.05)$ indicating slightly greater post-drug increases in these measures in the ADHD group $(B=-9.7, p<$ 0.0001 for $A D H D$ and $B=-6.2, p<0.0001$ for controls). However, there were no significant group differences in physiological measures at any particular nicotine dose, and these differences were not clinically significant.

\section{Subjective effects}

Figure 2 illustrates results from the Initial Reactions Questionnaire. Pleasant, Unpleasant, and Dizzy reactions all increased linearly with dose (see Supplementary Table S2).

After controlling for race, age, and sex, there were significant group and/or group $\times$ dose interactions across 2 of the 3 outcomes associated with self-reported Initial Reactions.

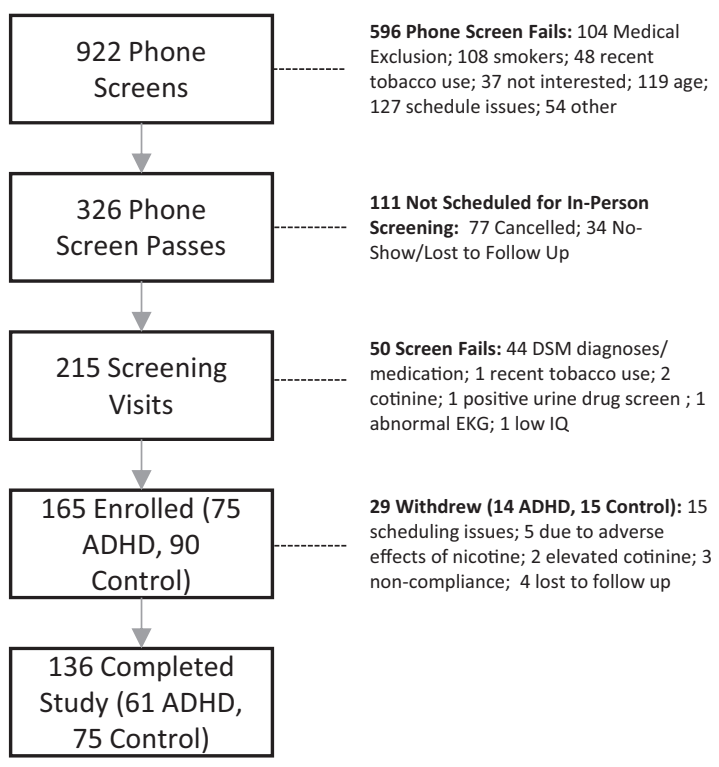

Fig. 1 Participant disposition through the study.
Table 2. Baseline demographic characteristics of ADHD and Control groups.

\begin{tabular}{|c|c|c|c|c|}
\hline & $\begin{array}{l}\text { ADHD } \\
\text { Mean }( \pm S D)\end{array}$ & $\begin{array}{l}\text { Control } \\
\text { Mean (and SD) }\end{array}$ & Test statistic & $p$ \\
\hline Age & $21.1(2.3)$ & $22.2(1.9)$ & $T=2.94$ & $* *$ \\
\hline$\%$ Female & $54 \%(n=33)$ & $49 \%(n=37)$ & $x^{2}=0.31$ & \\
\hline$\%$ Non-white & $34 \%(n=21)$ & $56 \%(n=42)$ & $x^{2}=6.30$ & $*$ \\
\hline Estimated IQ & $116.9(12.0)$ & $116.3(16.9)$ & $T=0.26$ & \\
\hline$\%$ College graduate & $33 \%(n=20)$ & $53 \%(n=40)$ & $x^{2}=5.76$ & $*$ \\
\hline \multicolumn{5}{|l|}{ CAARS subscale $T$-scores } \\
\hline Inattention & $77.6(10.1)$ & $42.6(7.9)$ & $T=22.68$ & $* * *$ \\
\hline Hyperactivity/impulsivity & $65.7(13.1)$ & $38.8(6.5)$ & $T=14.61$ & *** \\
\hline Total score & $76.1(10.2)$ & $39.6(8.0)$ & $T=23.37$ & $* * *$ \\
\hline$\%$ Previous ADHD diagnosis & $71 \%(n=43)$ & N/A & & \\
\hline \multicolumn{5}{|l|}{ ADHD subtype } \\
\hline $\begin{array}{l}\text { Predominantly } \\
\text { inattentive }\end{array}$ & $49 \%(n=30)$ & $\mathrm{N} / \mathrm{A}$ & & \\
\hline Combined & $51 \%(n=31)$ & $\mathrm{N} / \mathrm{A}$ & & \\
\hline Currently on medication & $31 \%(n=19)$ & & & \\
\hline
\end{tabular}

A significant Group $\times$ Dose interaction emerged for the Dizziness outcome $(B=0.40, p<0.05)$. The groups were comparable at the $0.0 \mathrm{mg}$ dose, but $A D H D$ participants reported significantly higher dizziness at the $0.5 \mathrm{mg}(B=0.21, p<0.05)$ and $1.0 \mathrm{mg}$ doses $(B=0.41, p<0.05)$. For Pleasant Reactions there was a main effects of group characterized by higher values across all three nasal spray doses for the ADHD compared to the Control group $(B=0.30,0.31$, and 0.32 , for $0.0,0.5$, and $1.0 \mathrm{mg}$ doses, respectively, all $\left.p^{\prime} s<0.005\right)$. Ratings of Unpleasant Reactions did not differ between groups.

A similar pattern of results was observed for the VAS used to measure other subjective drug effects (see Supplementary Table S2). Nicotine dose-dependently increased all of the subjective ratings $\left(B^{\prime} s>4.0, p^{\prime} s<0.01\right)$, with the exception of Would Like to Take Again. Significant main effects of group (ADHD > Control) emerged for ratings of Feel Drug, Good Effects, Like Drug, and Would Like to Take Again $\left(B^{\prime} s>7.3 ; p^{\prime} s<0.05\right)$. There were no main effects of group or group interactions for ratings of Bad Effects.

Nicotine choice

Figure 3 and Supplementary Table S3 illustrate results of the choice sessions as a function of group and cognitive condition. Controlling for age, sex, race, and order effects, there was a significant main effect of group $(B=0.53, p<0.05)$, as ADHD subjects made more choices for nicotine relative to controls. A main effect of cognitive condition was also observed $(B=0.45$, $p<0.01$ ), with more choices for nicotine made under high relative to low cognitive demand. Although the group by condition interaction was not significant, examination of simple effects indicated that $A D H D$ subjects chose significantly more nicotine than controls during the $\mathrm{LD}$ condition $(B=0.78, p<0.05)$, whereas the groups did not differ during the HD condition. Similarly, the effect of cognitive condition on choice was only significant within the control group, such that controls increased nicotine choices under high relative to low cognitive demand $(B=0.30, p<0.01)$. By contrast, ADHD subjects' generally greater choices for nicotine were not influenced by cognitive demand.

\section{DISCUSSION}

The current study demonstrates that nonsmoking young adults diagnosed with ADHD exhibit a different pattern of subjective and 

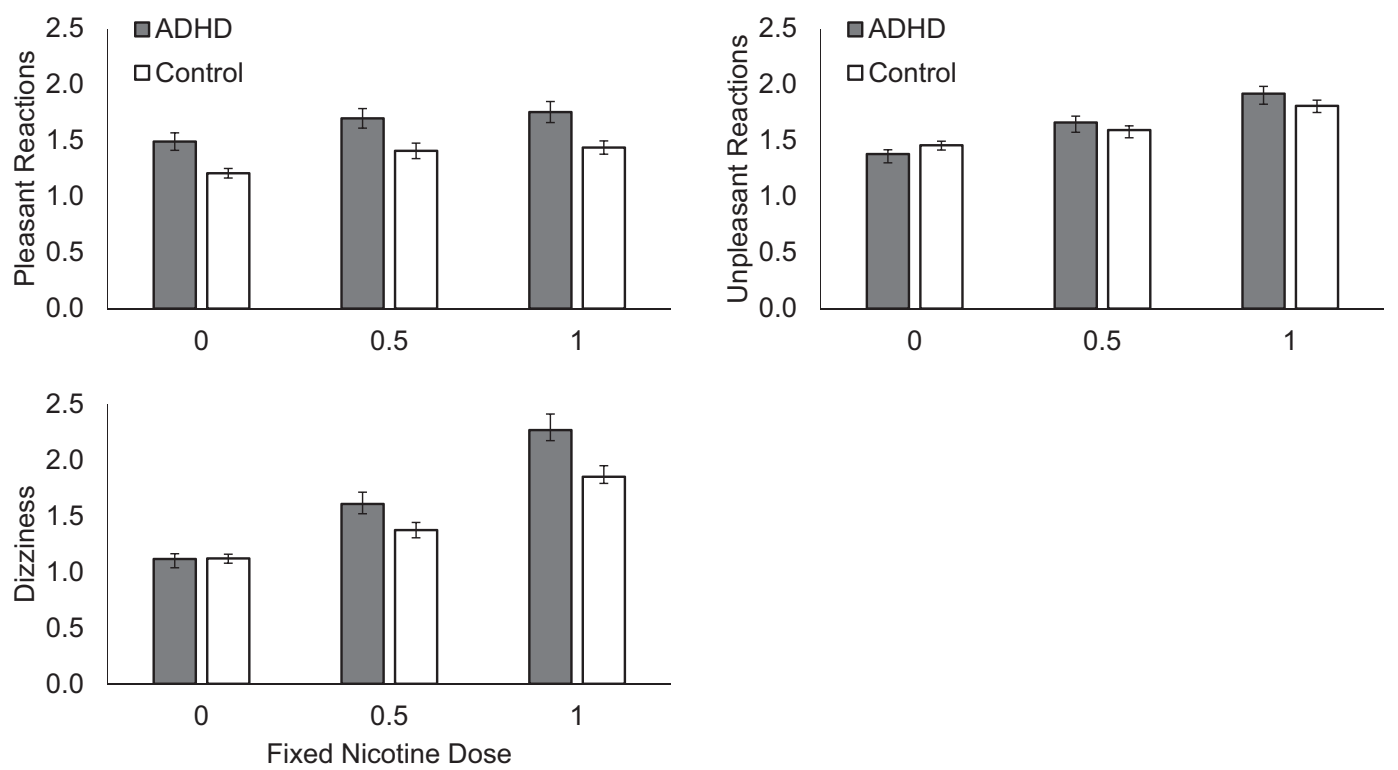

Fig. 2 Subjective effects of nicotine by group across doses. See text for statistical details.

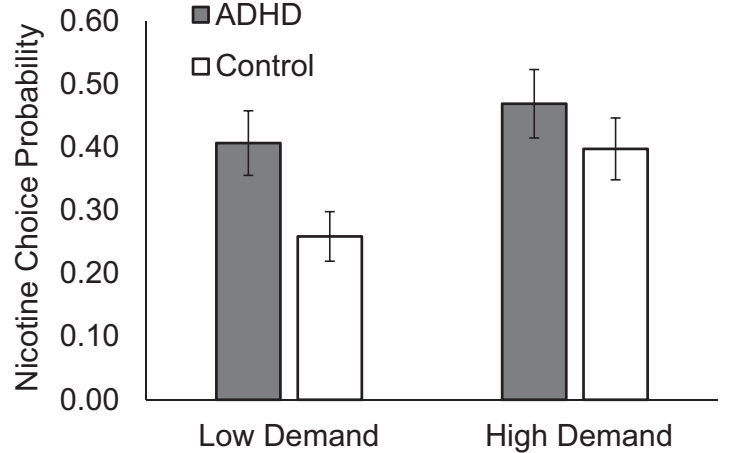

Fig. 3 Results from choice sessions by group and cognitive demand condition. See text for statistical details.

behavioral responses to acute nicotine exposure compared to peers without ADHD. Non-smokers with ADHD reported significantly greater dizziness effects of intranasal nicotine dose per se, and greater pleasant subjective effects across all nasal spray doses, compared to non-ADHD non-smokers, and there were no group differences on subjective reports of bad or unpleasant effects. Notably, rates of nicotine self-administration were significantly higher among non-smokers with ADHD, and their choices of nicotine were not influenced by cognitive condition. Comparatively greater nicotine choice by ADHD participants versus controls under the low demand condition (e.g. casual rest) may suggest higher nicotine reinforcement under conditions more representative of the natural environment than high demand [27]. As observed in other studies of nicotine naïve individuals, choice of nicotine spray was not preferred overall but varied among individuals [13].

A significant group by dose interaction was observed for selfreported dizziness, which does not load clearly onto positive or negative factors. This item is a robust predictor of subsequent smoking $[28,29]$, has a strong heritable component, and has been proposed as the clearest index of underlying nicotine sensitivity [30]. The observation that ADHD non-smokers' report of greater pleasant subjective effects was not moderated by nasal spray dose potentially indicates an increased positive response to novel experience (i.e., nasal spray) rather than effects of nicotine per se. Together with their greater choice of nicotine sprays, these results support the hypothesis that individuals with ADHD are more sensitive to initial exposure to nicotine, thereby placing them at greater risk for progression of smoking.

To our knowledge, however, this is the first experimental demonstration that a clinical group at risk for a particular type of substance use exhibits a different pattern of response to that substance during initial exposure. And while the magnitude of effects observed in the present study was modest, the findings have important implications for public health. Specifically, the results suggest that smoking/nicotine use prevention efforts for individuals with ADHD should be initiated prior to the age at which experimentation typically occurs (i.e. early adolescence), especially since it has been shown that the transition from initial experimentation to nicotine dependence is faster among individuals with high levels of ADHD symptoms [31].

Several limitations should be considered. First, for ethical and practical reasons, we studied young adults who had self-selected to being non-users of nicotine and could independently provide consent to participate. As such the degree to which our findings generalize to younger individuals remains unknown. This is important since it is known that individuals with ADHD or high levels of ADHD symptoms are likely to experiment with nicotine/ smoking at younger ages than those without such characteristics $[2,32]$. Second, as noted above, some outcomes, including pleasant subjective effects, showed main effects of ADHD status and not interactions with dose, suggesting that nicotine per se was not driving those group differences. These findings still have relevance for understanding smoking risk since initial experimentation involves novel physiological and sensory experiences. Finally, our study was conducted, for the most part, prior to the widespread use of novel electronic nicotine delivery systems (i.e., e-cigarettes). Given that a substantial and growing minority of youth report recent e-cig use [33], the relevance of our findings to experimentation and uptake of e-cigarette use is unknown, though the pharmacokinetic and sensory experiences of nicotine nasal spray may be more similar to e-cigarette use (i.e., both routes lack combustion) than cigarette smoking. Still, additional work is needed to clarify how the landscape of e-cigarette use influences nicotine/tobacco use and dependence among individuals with ADHD.

In spite of limitations, the current study used a large and wellcharacterized sample; as well as the use of well-validated behavioral pharmacological methods to evaluate both the subjective and 
reinforcing effects of nicotine. The present findings suggest that there are meaningful differences between young adults with and without ADHD with respect to their initial reactions to nicotine. And given the strong relationship between initial reactions and risk for regular use, interventions to prevent use should start prior to typical age of experimentation among ADHD patients.

\section{FUNDING AND DISCLOSURE}

This project was funded by R01DA033080 (PI: Kollins) and K24DA023464 (PI: Kollins). None of the authors reports disclosures relevant to this work. The authors declare no competing interests.

\section{ADDITIONAL INFORMATION}

Supplementary Information accompanies this paper at (https://doi.org/10.1038/ s41386-019-0581-7)

Publisher's note Springer Nature remains neutral with regard to jurisdictional claims in published maps and institutional affiliations.

\section{REFERENCES}

1. Kessler RC, Adler L, Barkley R, Biederman J, Conners CK, Demler O, et al. The prevalence and correlates of adult ADHD in the United States: results from the National Comorbidity Survey Replication. Am J Psychiatry. 2006;163:716-23.

2. Mitchell JT, Howard AL, Belendiuk KA, Kennedy TM, Stehli A, Swanson JM, et al. Cigarette smoking progression among young adults diagnosed with ADHD in childhood: a 16-year Longitudinal Study of Children with and without ADHD. Nicotine Tob Res 2019;21:638-47. https://doi.org/10.1093/ntr/nty045.

3. Visser SN, Danielson ML, Bitsko RH, Holbrook JR, Kogan MD, Ghandour RM, et al. Trends in the parent-report of health care provider-diagnosed and medicated attention-deficit/hyperactivity disorder: United States, 2003-11. J Am Acad Child Adolesc Psychiatry. 2014;53:34-46.e2.

4. Wilens $T E$, Vitulano $M$, Upadhyaya $H$, Adamson J, Sawtelle $R$, Utzinger $L$, et al. Cigarette smoking associated with attention deficit hyperactivity disorder. J Pediatr. 2008;153:414-9.

5. Wilens $T E$, Vitulano $M$, Upadhyaya $H$, Adamson J, Parcell $T$, Westerberg $D$, et al. Concordance between cigarette smoking and the modified Fagerstrom Tolerance Questionnaire in controlled studies of ADHD. Am J Addict. 2008;17:491-6.

6. Milberger S, Biederman J, Faraone SV, Chen L, Jones J. ADHD is associated with early initiation of cigarette smoking in children and adolescents. J Am Acad Child Adolesc Psychiatry. 1997;36:37-44.

7. Rohde P, Kahler CW, Lewinsohn PM, Brown RA. Psychiatric disorders, familial factors, and cigarette smoking: II. Associations with progression to daily smoking. Nicotine Tob Res. 2004;6:119-32.

8. Kollins SH, English JS, Roley ME, O'Brien B, Blair J, Lane SD, et al. Effects of smoking abstinence on smoking-reinforced responding, withdrawal, and cognition in adults with and without attention deficit hyperactivity disorder. Psychopharmacology. 2013;227:19-30.

9. McClernon FJ, Kollins SH, Lutz AM, Fitzgerald DP, Murray DW, Redman C, et al. Effects of smoking abstinence on adult smokers with and without attention deficit hyperactivity disorder: results of a preliminary study. Psychopharmacology. 2008;197:95-105.

10. McClernon FJ, Van Voorhees EE, English J, Hallyburton M, Holdaway A, Kollins SH. Smoking withdrawal symptoms are more severe among smokers with ADHD and independent of ADHD symptom change: results from a 12-day ContingencyManaged Abstinence Trial. Nicotine Tob Res 2011;13:784-92. https://doi.org/ 10.1093/ntr/ntr073.

11. Sweitzer MM, Kollins SH, Kozink RV, Hallyburton M, English J, Addicott MA, et al. ADHD, smoking withdrawal, and inhibitory control: results of a Neuroimaging Study with methylphenidate challenge. Neuropsychopharmacology. 2018;43:851-8.
12. Perkins KA, Sexton JE, Reynolds WA, Grobe JE, Fonte C, Stiller RL. Comparison of acute subjective and heart rate effects of nicotine intake via tobacco smoking versus nasal spray. Pharm Biochem Behav. 1994;47:295-9.

13. Perkins KA, Gerlach D, Broge M, Fonte $C$, Wilson A. Reinforcing effects of nicotine as a function of smoking status. Exp Clin Psychopharmacol. 2001;9:243-50.

14. Perkins KA, Lerman C, Coddington SB, Jetton C, Karelitz JL, Scott JA, et al. Initial nicotine sensitivity in humans as a function of impulsivity. Psychopharmacology. 2008;200:529-44.

15. Levin ED, McClernon FJ, Rezvani AH. Nicotinic effects on cognitive function: behavioral characterization, pharmacological specification, and anatomic localization. Psychopharmacology. 2006;184:523-39.

16. Javors MA, Hatch JP, Lamb RJ. Cut-off levels for breath carbon monoxide as a marker for cigarette smoking. Addiction. 2005;100:159-67.

17. First MB, Gibbon M, Spitzer RL, Williams JBW. User's guide for the Structured Clinical Interview for DSM-IV Axis I Disorders-research version. New York, NY: Biometrics Research Department; 1996.

18. Epstein JN, Johnson D, Conners CK. Conners' adult ADHD diagnostic interview for DSM-IV. North Tonawanda, NY: Multi-Health Systems, Inc; 2000.

19. Conners CK, Erhardt D, Sparrow E, staff MHS. The Conners Adult ADHD Rating Scale (CAARS). Toronto, Canada: Multi-Health Systems, Inc; 1998.

20. Kollins SH. Comparing the abuse potential of methylphenidate versus other stimulants: a review of available evidence and relevance to the ADHD patient. J Clin Psychiatry. 2003;64(Suppl 11):14-18.

21. Bidwell LC1, Garrett ME, McClernon FJ, Fuemmeler BF, Williams R, Ashley-Koch $A E$, et al. A preliminary analysis of interactions between genotype, retrospective ADHD symptoms, and initial reactions to smoking in a sample of young adults. Nicotine Tob Res. 2012;14:229-33. https://doi.org/10.1093/ntr/ntr125.

22. Hu MC, Davies M, Kandel DB. Epidemiology and correlates of daily smoking and nicotine dependence among young adults in the United States. Am J Public Health. 2006;96:299-308.

23. Pomerleau OF, Pomerleau CS, Namenek RJ. Early experiences with tobacco among women smokers, ex-smokers, and never-smokers. Addiction. 1998;93: 595-9.

24. Perkins KA, Grobe JE, Caggiula A, Wilson AS, Stiller RL. Acute reinforcing effects of low-dose nicotine nasal spray in humans. Pharm Biochem Behav. 1997;56: 235-41.

25. Liang KY, Zeger SL. A comparison of two bias-corrected covariance estimators for generalized estimating equations. Biometrika. 1986;73:13-22.

26. Wang M, Kong L, Li Z, Zhang L. Covariance estimators for generalized estimating equations (GEE) in longitudinal analysis with small samples. Stat Med. 2016; 35:1706-21

27. Bureau of Labor Statistics. Time spent in leisure activities in 2014 , by gender, age, and educational attainment. [online] 2019. Available at: https://www.bls.gov/ opub/ted/2015/time-spent-in-leisure-activities-in-2014-by-gender-age-andeducational-attainment.htm. Accessed 5 Nov 2019.

28. Pomerleau CS, Pomerleau OF, Namenek RJ, Marks JL. Initial exposure to nicotine in college-age women smokers and never-smokers: a replication and extension. J Addict Dis. 1999;18:13-19.

29. Zabor EC, Li Y, Thornton LM, Shuman MR, Bulik CM, Lichtenstein P, et al. Initial reactions to tobacco use and risk of future regular use. Nicotine Tob Res. 2013;15:509-17.

30. Haberstick BC, Ehringer MA, Lessem JM, Hopfer CJ, Hewitt JK. Dizziness and the genetic influences on subjective experiences to initial cigarette use. Addiction. 2011;106:391-9.

31. Fuemmeler BF, Kollins SH, McClernon FJ. Attention deficit hyperactivity disorder symptoms predict nicotine dependence and progression to regular smoking from adolescence to young adulthood. J Pediatr Psychol. 2007;32:1203-13.

32. Kollins SH, McClernon FJ, Fuemmeler BF. Association between smoking and attention-deficit/hyperactivity disorder symptoms in a population-based sample of young adults. Arch Gen Psychiatry. 2005;62:1142-7.

33. Cullen KA, Ambrose BK, Gentzke AS, Apelberg BJ, Jamal A, King BA. Notes from the field: use of electronic cigarettes and any tobacco product among middle and high school students-United States, 2011-2018. Morb Mortal Wkly Rep. 2018;67:1276-7. 